\title{
Propagation of Singularities in the Ramified Cauchy Problem for a Class of Operators with Non-involutive Multiple Characteristics
}

By

\author{
Katsuju IGARI*
}

\begin{abstract}
In the ramified Cauchy problem, analytic continuation of holomorphic solutions has been mainly studied. In this paper, we prove the propagation of singularities for a class of linear partial differential equations with non-involutive multiple characteristics.
\end{abstract}

\section{§1. Introduction}

In this paper, we consider the Cauchy problem in a complex domain with singular initial data and study the propagation of singularities of the solution.

It is one of the most fundamental problems in the theory of partial differential equations, and its study goes back to J. Leray [10]. Since then, many important articles have been published for this problem, especially $\mathrm{Y}$. Hamada, J. Leray and C. Wagschal [4] for operators with characteristics of constant multiplicity, D. Schiltz, J. Vaillant and C. Wagschal [14] and C. Wagschal [16] for operators with involutive multiple characteristics. There are also several interesting papers for operators with non-involutive multiple characteristics, e.g., J. Urabe [15]. C. Wagschal [17], J. Persson [13], S. Ouchi [12] and S. Fujiie [2]. In those papers, the analytic continuation of the holomorphic solution was mainly studied.

Consider the following Cauchy problem first.

$$
\left\{\begin{array}{l}
P^{(1)} u:=\left\{D_{1}^{2}-z_{1}^{2} D_{n}^{2}+\sum_{j=1}^{n} b_{j} D_{j}+c\right\}_{u}=0 \\
D_{1}^{i} u\left(0, z^{\prime}\right)=u_{i}\left(z^{\prime}\right), i=0,1
\end{array}\right.
$$

Communicated by T. Kawai, July 13, 1998. Revised October 22, 1998.

1991 Mathematics Subject Classification (s): 35A10, 35A20, 35B60

*Ehime University, Faculty of Engineering, Laboratory of Applied Mathematics, Matsuyama, 7908577, Japan

e-mail: igari@en1.ehimc-u.ac.jp 
where $z=\left(z_{1}, \cdots, z_{n}\right) \in \mathbb{C}^{n}, z^{\prime}=\left(z_{2}, \cdots, z_{n}\right), n \geq 2, D_{j}=\partial / \partial z_{j}$, the coefficients are all holomorphic in $\Omega_{r}:=\left\{z ;\left|z_{i}\right|<r_{i}, \forall i\right\}$ with $r=\left(r_{1}, \cdots, r_{n}\right), r_{i}>0$.

Denote $S:=\left\{z_{1}=0\right\}$ and $T:=\left\{z_{1}=z_{n}=0\right\}$, and suppose the initial data $u_{i}\left(z^{\prime}\right)$ are both holomorphic at a point $z^{\prime}=a^{\prime} \in(S-T) \cap \Omega$ and have a holomorphic extension in the universal covering space $\mathscr{R}\{(S-T) \cap \Omega\}$ each, that is, each $u_{i}\left(z^{\prime}\right)$ can be analytically continued along any path (continuous curve) in (ST) $\cap \Omega$ issued from $a^{\prime}$. Note $K^{1}:=\left\{z_{n}+z_{1}^{2} / 2=0\right\}$ and $K^{2}:=\left\{z_{n}-z_{1}^{2} / 2=0\right\}$ are characteristic surfaces of $P^{(1)}$ issued from $T$.

By the Cauchy-Kovalevskaya theorem, there is a unique holomorphic solution $u(z)$ to the Cauchy problem (1) in a neighborhood of $a=\left(0, a^{\prime}\right)$. Note one may replace the starting point $a=\left(0, a^{\prime}\right)$ with another one arbitrarily. Then it follows from the result of C. Wagschal [17] that there exists $\rho=\left(\rho_{1}, \cdots, \rho_{n}\right)$, $\rho_{i}>0$, such that the local holomorphic solution $u(z)$ at $a=\left(0, a^{\prime}\right) \in(S-T) \cap \Omega_{o}$ has a holomorphic extension in the universal covering space $\mathscr{R}\left(\Omega_{\rho}-K^{1} \cup K^{2}\right)$. (J. Persson [13] proved it with a different method. J. Urabe [15] gave an expression of the solution in terms of hypergeometric functions when the initial data are meromorphic and $b_{n}\left(0, z^{\prime}\right)$ is constant.)

Now, let us say $\widehat{z} \in K^{1} \cup K^{2}$ is a weak singular point of $u$ if there is a path issued from $a$ and traced in $\Omega_{\rho}-K^{1} \cup K^{2}$ except the end point $\widehat{z}$ along which $u(z)$ can not be analytically continued up to $\widehat{z}$. (See Definition 2.1 .) Then one of our aims in this article is to investigate the equality

$$
\text { w.sing.supp. }[u]=\left(K^{1} \cup K^{2}\right) \cap \Omega_{\rho}
$$

where w.sing.supp. $[u]$ denotes the weak singular support of $u$ in the above sense. This equality is a complex version of the branching of singularities of solutions in real domains (cf. S. Alinhac [1], N. Hanges [5] and T. Oaku [11]).

Consider the following Cauchy problem as well.

$$
\left\{\begin{array}{l}
P^{(2)} u:=\left\{z_{1} D_{1}^{2}-z_{1} D_{n}^{2}+\sum_{j=1}^{n} b_{j} D_{j}+c\right\}_{u}=0 \\
u\left(0, z^{\prime}\right)=u_{0}\left(z^{\prime}\right)
\end{array}\right.
$$

where the coefficients are all holomorphic in $\Omega_{r}$ and the initial datum $u_{0}\left(z^{\prime}\right)$ is holomorphic at a point $z^{\prime}=a^{\prime} \in(S-T) \cap \Omega$ and has a holomorphic extension in the universal covering space $\mathscr{R}\{(S-T) \cap \Omega\}$.

The operator $P^{(2)}$ is of Fuchs type in the sense of Baouendi-Goulaouic. $K^{1}:=\left\{z_{n}+z_{1}=0\right\}$ and $K^{2}:=\left\{z_{n}-z_{1}=0\right\}$ are characteristic surfaces issued from $T$, and the initial surface $S$ is also characteristic. If $b_{1}(0) \neq 0,-1,-2, \cdots$, there is a unique holomorphic solution $u(z)$ to the Cauchy problem (2) in a neighborhood of $a=\left(0, a^{\prime}\right)$ by the Cauchy-Kovalevskaya type theorem due to $\mathrm{Y}$. Hasegawa [6]. Moreover, it follows from S. Ouchi [12] that there exists $\rho=\left(\rho_{1}\right.$, $\left.\cdots, \rho_{n}\right), \rho_{i}>0$, such that this local holomorphic solution $u(z)$ at $a=\left(0, a^{\prime}\right) \in(S-$ $T) \cap \Omega_{\rho}$ has a holomorphic extension in the universal covering space $R\left(\Omega_{\rho}-S \cup\right.$ 
$K^{1} \cup K^{2}$ ). (Cf. S. Fujiie [2], too.) It is also our aim to investigate how the singularities of the initial datum propagate in this case.

By the way, the operators $P^{(1)}$ and $P^{(2)}$ have a common property. Denote $\lambda^{1}=z_{1} \zeta_{n}, \lambda^{2}=-z_{1} \zeta_{n}, a=1$ for $P^{(1)}$, and $\lambda^{1}=\zeta_{n}, \lambda^{2}=-\zeta_{n}, a=z_{1}$ for $P^{(2)}$. Let $P_{2}$ denote their principal part. Then $P^{(1)}$ and $P^{(2)}$ both satisfy the following condition.

Condition $\mathbf{N}$. There are $\lambda^{i}\left(z, \zeta^{\prime}\right), i=1,2$, holomorphic in a neighborhood of $\left(z, \zeta^{\prime}\right)=\left(0, \nu^{\prime}\right)$ such that the following 1$\left.)-3\right)$ hold:

1) $P_{2}(z, \zeta)=a(z)\left\{\zeta_{1}-\lambda^{1}\left(z, \zeta^{\prime}\right)\right\}\left\{\zeta_{1}-\lambda^{2}\left(z, \zeta^{\prime}\right)\right\}$

2) $Q^{1}\left(z, \zeta^{\prime}\right):=\partial P_{2} /\left.\partial \zeta_{1}\right|_{\zeta_{1}=\lambda^{1}\left(z, \zeta^{\prime}\right)}=0$ on $\left\{z_{1}=0, \zeta^{\prime}=\nu^{\prime}\right\}$

3) $A^{1}:=\left\{\zeta_{1}-\lambda^{1}\left(z, \zeta^{\prime}\right), a(z)\left(\zeta_{1}-\lambda^{2}\left(z, \zeta^{\prime}\right)\right)\right\}_{\zeta_{1}=\lambda^{1}\left(z, \zeta^{\prime}\right),\left(z, \zeta^{\prime}\right)=\left(0, \nu^{\prime}\right)} \neq 0$

where $a(z)$ is the coefficient of $D_{1}^{2}, \nu^{\prime}=(0, \cdots, 0,1) \in \mathbf{C}^{n-1}$ and $\{, \quad\}$ stands for the Poisson bracket.

In this paper we generally study the operators satisfying this condition.

In Section 2, the main results will be stated. The main theorem (Theorem 2.2 ) is on removable singularities of solutions of $P u=0$, where $P$ is an arbitrary operator satisfying the above condition. The problems mentioned above will be answered for more general operators by its corollaries (Corollaries 2.3 and 2.4).

The main theorem will be proved in Sections 3 and 4. Hartogs' method, Cauchy-Kovalevskaya type theorem obtained in our preceding paper [8] and Zerner's method [18] are essentially used there. It is remarkable that there are three standard forms of the operators satisfying the above condition (Proposition 4.2).

In Section 5, an extension of the main theorem to higher order operators will be given.

The following notations are employed in this article:

$$
\begin{array}{ll}
n \geq 2, & z=\left(z_{1}, \cdots, z_{n}\right), \\
z^{\prime}=\left(z_{2}, \cdots, z_{n}\right), & D_{j}=\partial / \partial z_{j}, \\
D=\left(D_{1}, \cdots, D_{n}\right), & D^{\prime}=\left(D_{2}, \cdots, D_{n}\right), \\
r=\left(r_{1}, \cdots, r_{n}\right), r_{i}>0 & \Omega=\Omega_{r}=\left\{z ;\left|z_{i}\right|<r_{i}, \forall i\right\}, \\
S=\left\{z_{1}=0\right\}, & T=\left\{z_{1}=z_{n}=0\right\}, \\
R(\cdot): \text { universal covering space of } \cdot
\end{array}
$$

\section{§2. Statement of the Main Results}

\subsection{Main Theorem}

Let $P=P(z, D)$ be a linear partial differential operator of 2 nd order with 
holomorphic coefficients in $\Omega=\Omega_{r}$ and its principal symbol $P_{2}(z, \zeta),(z, \zeta) \in \Omega$ $\times \mathbb{C}^{n}$, satisfy the Condition $\mathrm{N}$ stated in Introduction.

Denote by $\Phi^{i}(z)$ the solution of the Cauchy problem

$$
D_{1} \Phi-\lambda^{i}\left(z, D^{\prime} \Phi\right)=0, \Phi\left(0, z^{\prime}\right)=z_{n} .
$$

(Since $D^{\prime} \Phi(0)=\nu^{\prime}$, the Cauchy Kovalevskaya theorem applies to this problem.) By the implicit function theorem, taking $r=\left(r_{1}, \cdots, r_{n}\right)$ appropriately, one may suppose that the equation $\Phi^{i}(z)=0$ is solved by $z_{n}=\varphi^{i}\left(z_{1}, \cdots, z_{n-1}\right)$ in $\Omega_{r}$ with a holomorphic function $\varphi^{i}$ in $\left\{\left|z_{j}\right|<r_{j}, \forall j \leq n-1\right\}$ satisfying $\varphi^{i}\left(0, z_{2}, \cdots, z_{n-1}\right)=0$ and $\left|\varphi^{i}\left(z_{1}, \cdots, z_{n-1}\right)\right|<r_{n}$.

Denote $K^{i}=\left\{z_{n}=\varphi^{i}\left(z_{1}, \cdots, z_{n-1}\right)\right\}$. They are characteristic surfaces of $P$ issued from $T$. By replacing $\left(r_{1}, \cdots, r_{n-1}\right)$ with smaller ones if necessary, one may also suppose $K^{1} \cap K^{2}=T$. (See Section 4, 4.7.)

Let $A^{1}$ be the constant appearing in 3) of Condition $\mathrm{N}$ and set $A^{2}=-A^{1}$. Besides, set $B^{i}=P \Phi^{i}(0)$. We call $F^{i}(\mu):=A^{i} \mu+B^{i}$ the indicial polynomial of $P$ with respect to the characteristic surface $K^{i}$, and its zero the characteristic index. We assume the so-called indicial condition

$$
F^{i}(\mu) \neq 0, \forall \mu \in\{0,1,2, \cdots\}, i=1,2 .
$$

Definition 2.1. Let $V$ be an open connected set in $\mathbf{C}^{n}, \partial V$ stand for the boundary of $V, u(z)$ be holomorphic at a point $z^{\circ} \in V$ and it have a holomorphic extension in the universal covering space $\mathscr{R}(V)$. We say $\widehat{z} \in \partial V$ is a point of strong (weak) analytic continuation of $u(z)$, if $u(z)$ is analytically continued along any path (some path respectively) $z=z(t), 0 \leq t \leq 1$, satisfying

$$
z(0)=z^{\circ}, \quad z(1)=\widehat{z}, \quad z(t) \in V \text { for } t \neq 1 .
$$

If not, we call it a weak (strong respectively) singular point of $u$.

Theorem 2.2 (Main theorem)。 Suppose the Condition $N$ and the indicial condition (3). Let $V=\Omega-S \cup K^{1} \cup K^{2}, u(z)$ be a function holomorphic in a neighborhood of a point $z^{\circ} \in V$, satisfy the equation $P u=0$ and have a holomorphic extension in the universal covering $\mathscr{R}(V)$. Then, if $u(z)$ has a point of strong analytic continuation $\widehat{z} \in\left(K^{1} \cup K^{2}\right) \cap \Omega$ and a point of weak one a $\in S \cap \Omega$, it has a unique holomorphic extension in $\Omega$. (Removable singularities)

This theorem will be proved in Sections 3 and 4 .

\subsection{Standard Forms of the Operators}

Consider the following three operators.

$$
P^{A}:=a(z)\left[D_{1}^{2}-\left\{\sum_{j=2}^{n-1} a_{j}(z) D_{j}+z_{1} a_{n}(z) D_{n}\right\}^{2}\right]+\sum_{j=1}^{n} b_{j}(z) D_{j}+c(z)
$$




$$
\begin{aligned}
& P^{B}:=a(z)\left\{D_{1}^{2}-z_{1}^{2} \sum_{j, k=2}^{n} a_{j k}(z) D_{j} D_{k}\right\}+\sum_{j=1}^{n} b_{j}(z) D_{j}+c(z) \\
& P^{C:}=z_{1} a(z)\left\{D_{1}^{2}-\sum_{j, k=2}^{n} a_{j k}(z) D_{j} D_{k}\right\}+\sum_{j=1}^{n} b_{j}(z) D_{j}+c(z)
\end{aligned}
$$

where all the coefficients are holomorphic, $a(0) \neq 0, a_{n}(0) \neq 0$ and $a_{n n}(0) \neq 0$ in every case.

$P^{(1)}$ is a special case of $P^{A}$ and of $P^{B}$, and $P^{(2)}$ is a special one of $P^{C}$. It is easy to see that these three operators satisfy Condition N. Conversely, we will see that any operator satisfying Condition $\mathrm{N}$ can be reduced to one of these three operators by a suitable change of variables (Proposition 4.2).

We will also see the indicial conditions of $P^{A}, P^{B}$ and $P^{C}$ are respectively written in terms of their coefficients as follows.

$$
\begin{aligned}
& F_{A}^{ \pm}:= \pm a(0) a_{n}(0)\{2 \mu+1\}+b_{n}(0) \neq 0 \quad \forall \mu \in\{0,1,2, \cdots\}, \\
& F_{B}^{ \pm}:= \pm a(0) \sqrt{a_{n n}(0)}\{2 \mu+1\}+b_{n}(0) \neq 0 \quad \forall \mu \in\{0,1,2, \cdots\} \text {, } \\
& F_{c}^{ \pm}:= \pm \sqrt{a_{n n}(0)}\left\{2 a(0) \mu+b_{1}(0)\right\}+b_{n}(0) \neq 0 \quad \forall \mu \in\{0,1,2, \cdots\} \text {. }
\end{aligned}
$$

(See Section 4, 4.7.)

\subsection{Propagation of Singularities}

For $P=P^{A}$ or $P^{B}$, consider the Cauchy problem

$$
P u=0, \quad D^{i} u\left(0, z^{\prime}\right)=u_{i}\left(z^{\prime}\right), \quad i=0,1
$$

where $u_{i}\left(z^{\prime}\right)$ are holomorphic at a point $a^{\prime} \in(S-T) \cap \Omega$ and have a holomorphic extension in the universal covering $\mathscr{R}\{(S-T) \cap \Omega\}$ each.

Under the corresponding indicial condition, the following corollary directly follows from Theorem 2.2. It is a complex version of the branching of singularities in real domains (Cf. [1], [5] and [11]) and gives an answer to the first problem mentioned in Introduction.

Corollary 2.3. Let $V=\Omega-K^{1} \cup K^{2}, u(z)$ be the local holomorphic solution of (5) in a neighborhood of $a=\left(0, a^{\prime}\right) \in(S-T) \cap \Omega$ and have a holomorphic extension in the universal covering $\mathscr{R}(V)$. Then, if $\bigcup_{i=0,1}$ sing.supp. $\left[u_{i}\left(z^{\prime}\right)\right] \neq \emptyset$, it holds that

$$
\text { w.sing.supp. }[u]=\left(K^{1} \cup K^{2}\right) \cap \Omega
$$

where w.sing.supp. [u] denotes the set of weak singular points of $u$ in the sense of Definition 2.1.

Remark. When $u_{i}\left(z^{\prime}\right)$ is holomorphic in $\mathscr{R}\{(S-T) \cap \Omega\}$, a weak singular 
point $\widehat{z}^{\prime} \in T$ is a strong singular point (cf. Proposition 3.3), so we need not distinguish these two types of singularities for initial data.

For $P=P^{C}$, consider the Cauchy problem

$$
P u=0, \quad u\left(0, z^{\prime}\right)=u_{0}\left(z^{\prime}\right),
$$

where $u_{0}\left(z^{\prime}\right)$ is holomorphic at a point $a^{\prime} \in(S-T) \cap \Omega$ and has a holomorphic extension in the universal covering $\mathscr{R}\{(S-T) \cap \Omega\}$. Under the corresponding indicial condition, we also have the following corollary. It gives an answer to the second problem stated in Introduction.

Corollary 2.4. Let $V=\Omega-S \cup K^{1} \cup K^{2}, u(z)$ be a local holomorphic solution of (6) in a neighborhood of $a=\left(0, a^{\prime}\right) \in(S-T) \cap \Omega$ and it have a holomorphic extension in the universal covering $\mathscr{R}(V)$. Then, if sing.supp. $\left[u_{0}\left(z^{\prime}\right)\right] \neq \emptyset$, it holds that

$$
\text { w.sing.supp. }[u] \supseteq\left(K^{1} \cup K^{2}\right) \cap \Omega \text {. }
$$

Remark. The assumption that $u(z)$ is holomorphic at $a=\left(0, a^{\prime}\right) \in(S-T) \cap$ $\Omega$ and has a holomorphic extension in the universal covering $\mathscr{R}(V)$ is equal to say that $u(z)$ is holomorphic at a point $z^{\circ} \in V$, has a holomorphic extension in the universal covering $\mathscr{R}(V)$ and has a point of weak analytic continuation $a=$ $\left(0, a^{\prime}\right) \in(S-T) \cap \Omega$.

\section{§3. Hartog's Method}

In this section, we prepare several propositions on the analytic continuation of holomorphic functions to prove the Main theorem.

Let $0<a^{\prime}{ }_{i}<a_{\imath}(i=1, \cdots, n)$ and denote $G:=\left\{z ;\left|z_{i}\right|<a_{i}, \forall i\right\}$ and $H:=\left\{\left|z_{\imath}\right|<a_{i}^{\prime} \forall\right.$ $\left.i \leq n-1,\left|z_{n}\right|<a_{n}\right\} \cup\left\{\left|z_{i}\right|<a_{i} \forall i \leq n-1, a_{n}^{\prime}<\left|z_{n}\right|<a_{n}\right\}$. The following theorem is due to Hartogs and fundamental in the theory of several complex variables.

Theorem 3.1 [Hartogs. Cf. [3]]. Every holomorphic function $u(z)$ in $H$ has a unique holomorphic extension in $G$.

Let $\widehat{z}=\left(\widehat{z}_{1}, \cdots, \widehat{z}_{n}\right)$ and denote $G_{\widehat{z}, a}:=\{z ; z-\widehat{z} \in G\}$ and $H_{\widehat{z}, a, a^{\circ}}:=\{z ; z-\widehat{z} \in H\}$, where $a=\left(a_{1}, \cdots, a_{n}\right)$ and $a^{\prime}=\left(a_{1}^{\prime}, \cdots, a_{n}^{\prime}\right)$. Theorem 3.1 holds for $\left\{G_{\widehat{z}, a}, H_{\hat{z}, a a^{\prime}}\right\}$, too. The pair $\left\{G_{\widehat{z}, a}, H_{\widehat{z}, a, a}\right\}$ is called a (euclidian) Hartogs figure.

Next, let $\rho_{i}>0(i=1, \cdots, n)$ and denote $U:=\left\{z ;\left|z_{i}\right|<\rho_{i}, \forall i\right\}$. Let $\varphi\left(z_{1}, \cdots, z_{n-1}\right)$ be a holomorphic function defined in $\left\{\left|z_{i}\right|<\rho_{i}, i \leq n-1\right\}$ with $\left|\varphi\left(z_{1}, \cdots, z_{n-1}\right)\right|<\rho_{n}$ and denote $K:=\left\{z_{n}=\varphi\left(z_{1}, \cdots, z_{n-1}\right)\right\}$.

Proposition 3.2 [Cf. [3, Chap. II, Th.1.5.]]. Let $\omega$ be a neighborhood of a point $\widehat{z} \in K$. Every holomorphic function $u(z)$ in $(U-K) \cup \omega$ has a holomorphic 
extension in $U$.

Proof. Let $z^{*}$ be an arbitrary point on $K$ and $\gamma: z=z(t), 0 \leq t \leq 1$, be a path on $K$ with $z(0)=\widehat{z}$ and $z(1)=z^{*}$. Then one can easily see by using Theorem 3.1 finite times that $u(z)$ is continued analytically along $\gamma$ up to $z^{*}$.

(Q.E.D.)

The following proposition is a simple modification of the above one.

Proposition 3.3. Suppose $u(z)$ is holomorphic at a point $z^{\circ} \in(U-K)$ and has a holomorphic extension in the universal covering space $\mathscr{R}(U-K)$. Then, if $u(z)$ has a point of weak analytic continuation $\widehat{z} \in K$, it has a holomorphic extension in $U$.

Proof. $u(z)$ is holomorphic in a neighborhood $\omega$ of $\widehat{z} \in K$ and it is easy to see that any closed path in $U-K$ is homotope with a path in $\omega-K$. Hence $u(z)$ must be single valued and one can apply Proposition 3.2.

(Q.E.D.)

Let $\Omega=\left\{z ;\left|z_{i}\right|<r_{i}, \forall i\right\}, r_{i}>0$ and $\varphi^{i}\left(z_{1}, \cdots, z_{n-1}\right), i=1,2$, be holomorphic functions in $\left\{\left(z_{1}, \cdots, z_{n-1}\right) ;\left|z_{i}\right|<r_{i}, i \leq n-1\right\}$ satisfying $\left|\varphi^{i}\left(z_{1}, \cdots, z_{n-1}\right)\right|<r_{n}, \varphi^{i}=0$ on $z_{1}=0$, and $\varphi^{1} \neq \varphi^{2}$ for $z_{1} \neq 0$. Denote $K^{i}=\left\{z_{n}=\varphi^{i}\left(z_{1}, \cdots, z_{n-1}\right)\right\}$.

Proposition 3.4. Let $V=\Omega-S \cup K^{1} \cup K^{2}$ and suppose $u(z)$ is holomorphic at a point $z^{\circ} \in V$ and has a holmorphic extension in the universal covering $\mathscr{R}(V)$. Then the following (a), (b) and (c) are equivalent:

(a) There is a point of strong analytic continuation $\widehat{z} \in\left(K^{2}-T\right) \cap \Omega$ of $u(z)$.

(b) Every $z^{*} \in\left(K^{2}-T\right) \cap \Omega$ is a point of strong analytic continuation of $u(z)$.

(c) $u(z)$ has a holomorphic extension in the universal covering $\mathscr{R}\left(\Omega-S \cup K^{1}\right)$.

Proof. We first prove $(\mathrm{a}) \Rightarrow(\mathrm{b})$. Let $z^{*}$ be an arbitrary point in $K^{2}-T$ and let us prove that $u(z)$ can be analytically continued up to $z^{*}$ along any path $\gamma$ : $z=z(t) \quad(0 \leq t \leq 1)$ satisfying $z(0)=z^{\circ}, z(1)=z^{*}$ and $z(t) \in V$ for $t \neq 1$.

Since $K^{2}-T$ is connected, there exists a path $\delta: z=\zeta(t)(0 \leq t \leq 1)$ such that $\zeta(0)=\widehat{z}, \zeta(1)=z^{*}$ and $\zeta(t) \in\left(K^{2}-T\right)$ for all $t$.

Let $0<\varepsilon<1$ and set $\zeta_{i}^{\varepsilon}(t):=\zeta_{i}(t)+\left\{z_{i}(1-\varepsilon)-\zeta_{i}(1)\right\} t$ for $i<n$, $\widetilde{\zeta}_{n}:=$ $\varphi \circ\left(\zeta_{1}^{\varepsilon}, \cdots, \zeta_{n-1}^{\varepsilon}\right)$ with $\varphi=\varphi^{2}$ and $\zeta_{n}^{\varepsilon}(t):=\widetilde{\zeta}_{n}^{\varepsilon}(t)+\left\{z_{n}(1-\varepsilon)-\widetilde{\zeta}_{n}^{\varepsilon}(1)\right\}_{t}$. Define a path $\delta^{\varepsilon}$ by $z=\zeta^{\varepsilon}(t)(0 \leq t \leq 1)$. Clearly, $\zeta^{\varepsilon}(0)=\widehat{z}, \zeta^{\varepsilon}(1)=z(1-\varepsilon)$, and if we take $\varepsilon$ sufficiently small, $\zeta^{\varepsilon}(t) \in V$ except for $t=0$.

Prolong $u(z)$ along $\gamma$ up to $z(1-\varepsilon)$ firstly. Since $\widehat{z}$ is a point of strong analytic continuation of $u$, one can successively prolong it up to $\widehat{z}$ along $-\delta^{\varepsilon}: z$ $=\zeta^{\varepsilon}(1-t),(0 \leq t \leq 1)$. The function element of $u$ at $\widehat{z}$ does not depend on $\varepsilon$.

By the way, there exist $\rho_{i}>0(1 \leq i \leq n)$ such that for any $t, U_{t}:=\left\{z ; \mid z_{i}-\right.$ $\left.\zeta_{i}(t) \mid<\rho_{i}\right\} \subset\left(\Omega-S \cup K^{1}\right)$ and $\left|\varphi\left(z_{1}, \cdots z_{n-1}\right)-\zeta_{n}(t)\right|<\rho_{n}$ for $\left\{\left|z_{i}-\zeta_{i}(t)\right|<\rho_{i}\right.$; $1 \leq i \leq n-1\}$. Besides, there is a sequence $0=t_{0}<t_{1}<\cdots<t_{N}=1$ such that $\zeta(t) \in$ $U_{t_{i}}$ for $t \in\left[t_{i}, t_{i+1}\right]$. Moreover, if $\varepsilon$ is sufficiently small, we have $\zeta^{\varepsilon}(t) \in U_{t_{i}}$ for $t \in\left[t_{i}, t_{i+1}\right]$, too. 
By Proposition 3.3, $u(z)$ has a holomorphic extension in $U_{t_{0}}$, which is at the same time a direct extension of $u$ at $\zeta^{\varepsilon}\left(t_{1}\right)$. Recurrently we see $u(z)$ at $\zeta\left(t_{i}\right)$ has a holomorphic extension in $U_{t_{t}}$, which is a direct extension of $u(z)$ at $\zeta^{\varepsilon}\left(t_{i+1}\right)$. Therefore $u(z)$ at $\zeta^{\varepsilon}\left(t_{N}\right)=z(1-\varepsilon)$ has a holomorphic extension in $U_{t_{N-1}}$. Since $z^{*} \in U_{t_{N-1}}$ and $z(t) \in U_{t_{N-1}}$ for $t \in[1-\varepsilon, 1]$ if $\varepsilon$ is sufficiently small, it means $u(z)$ is analytically continued along $\gamma$ up to $z^{*}$. Thus we have proved (b).

Next we prove $(\mathrm{b}) \Rightarrow(\mathrm{c})$. Let $\gamma: z=z(t)(0 \leq t \leq 1)$ be an arbitrary path satisfying $z(0)=z^{\circ}$ and $z(t) \in\left(\Omega-S \cup K^{1}\right)$. We show $u$ can be analytically continued up to $z(1)$ along $\gamma$.

Set $J:=\left\{t ; z(t) \in K^{2}-T\right\}$. If $J=\emptyset$, there are nothing to prove. So, we suppose $J \neq \emptyset$. Clearly there are $\rho_{i}>0(1 \leq i \leq n)$ such that for any $t \in J, U_{t}:=\{z$; $\left.\left|z_{i}-z_{i}(t)\right|<\rho_{i}\right\} \subset\left(\Omega-S \cup K^{1}\right)$ and $\left|\varphi\left(z_{1}, \cdots, z_{n-1}\right)-z_{n}(t)\right|<\rho_{n}$ for $\left\{\left|z_{i}-z_{i}(t)\right|<\rho_{i}\right.$; $1 \leq i \leq n-1\}$. Besides there exists a positive constant $\delta$ such that $\mid z_{i}(t)-z_{i}(\tau)$ $\mid<\rho_{i}$ for every $i$, every $\tau \in J$ and every $t \in[\tau-\delta, \tau+\delta] \cap[0,1]$.

Let $\tau_{0}:=\min \{\tau \in J\}$. Since $z\left(\tau_{0}\right) \in\left(K^{2}-T\right)$ is a point of strong analytic continuation of $u$ and $z(t)$ touches $K^{2}$ firstly at $t=\tau_{0}, u(z)$ can be analytically continued up to $z\left(\tau_{0}\right)$ along $\gamma$. By Proposition 3.3, $u(z)$ at $z\left(\tau_{0}\right)$ has a unique holomorphic extension in $U_{\tau_{0}}$. It means that $u$ can be continued along $\gamma$ up to $z(t)$ with $t=\min \left\{1, \tau_{0}+\delta\right\}$. If $\tau_{0}+\delta \geq 1$, the proof is complete. But, if $\tau_{0}+\delta<1$, we need to prolong $u$ beyond $z\left(\tau_{0}+\delta\right)$.

Denote $\tau_{1}=\max \left\{0, \tau_{0}-\delta\right\}$ and $\tau_{2}=\tau_{0}+\delta$. Since $U_{\tau_{0}}-K^{2}$ is connected and $z\left(\tau_{1}\right) \in\left(U_{\tau_{0}}-K^{2}\right)$, there is a path $z=z^{*}(t)$ in the interval $\left[\tau_{1}, \tau_{2}\right]$ such that

$$
\begin{aligned}
& z^{*}\left(\tau_{i}\right)=z\left(\tau_{i}\right), \quad i=1,2, \\
& z^{*}(t) \in\left(U_{\tau_{0}}-K^{2}\right), \quad \tau_{1} \leq t<\tau_{2},
\end{aligned}
$$

even if $z\left(\tau_{2}\right) \in K^{2}$. Let $\tilde{\gamma}$ denote the modified path of $\gamma$ with $z=z^{*}(t)$ in the interval $\left[\tau_{1}, \tau_{2}\right]$.

The analytic continuation along $\gamma$ and that along $\tilde{\gamma}$ give same function element at $z\left(\tau_{2}\right)$. Set $\widetilde{J}:=\left\{t ; \widetilde{z}(t) \in K_{2}\right\}$. If $\widetilde{J} \neq \emptyset$, denote $\widetilde{\tau}_{0}:=\min \{t \in \widetilde{J}\}$. Because $\widetilde{z}\left(\widetilde{\tau}_{0}\right)=z\left(\widetilde{\tau}_{0}\right)$ is a point of strong analytic continuation of $u$ and $\widetilde{z}(t)$ touches $K^{2}$ firstly at $t=\widetilde{\tau}_{0}$, it is possible to prolong $u$ along $\tilde{\gamma}$ (consequently along $\gamma$ as well) up to $z\left(\widetilde{\tau}_{0}\right)$. Note $\widetilde{\tau}_{0}-\tau_{0} \geq \delta$ and $\delta$ is independent of $\tau \in J$. Then we see, by repreating the above reasoning finite times, $u$ can be analytically continued along $\gamma$ up to $z(1)$. Thus (c) has been proved.

(Q.E.D.)

One can prove the following proposition exactly in the same way.

Proposition 3.5. Let $V=\Omega-K^{1} \cup K^{2}$ and suppose $u(z)$ is holomorphic at $z^{\circ}$ $\in V$ and has a holomorphic extension in $\mathscr{R}(V)$. Then, if $u(z)$ has a point of strong analytic continuation $\widehat{z} \in\left(K^{2}-T\right) \cup \Omega$, it has a holomorphic extension in $\mathscr{R}\left(\Omega-K^{1}\right)$. 
The following proposition seems similar to the above one, but there is an essential difference between them.

Proposition 3.6. Let $V=\Omega-S \cup K, u(z)$ be holomorphic at $z^{\circ} \in V$ and have a holomorphic extension in the universal covering space $\mathscr{R}(V)$. Then, if $u(z)$ has a point of weak analytic continuation $\widehat{z} \in(S-T) \cap \Omega$, it has a unique holomorphic extension in $\mathscr{R}(\Omega-K)$.

Proof. Since $\widehat{z}$ is a point of weak analytic continuation, there is a path $\gamma: z=$ $\zeta(t), 0 \leq t \leq 1$, satisfying

$$
\zeta(0)=z^{\circ}, \zeta(1)=\widehat{z}, \zeta(t) \in V \text { for } t \neq 1
$$

along which $u(z)$ is analytically continued up to $\widehat{z}$.

Let $\tilde{\gamma}: z=\tilde{\zeta}(t), 0 \leq t \leq 1$, be another arbitrary path satisfying (7) and set $\delta:=-\gamma+\widetilde{\gamma}$. One can write $\delta: z=z(t), 0 \leq t \leq 1$, by defining $z(t)=\zeta(1-2 t)$ for 0 $\leq t \leq 1 / 2$ and $z(t)=\widetilde{\zeta}(2 t-1)$ for $1 / 2 \leq t \leq 1$. It satisfies

$$
z(0)=z(1)=\widehat{z}, z(t) \in V \text { for } t \neq 0,1 .
$$

One can easily see there is a 1-parameter group of paths $\gamma^{\nu}: z=z^{\nu}(t), 0 \leq t$ $\leq 1$, being continuous in $\{0 \leq \nu \leq 1,0 \leq t \leq 1\}$ such that $z^{1}(t) \equiv z(t), z^{\nu}(t)$ satisfies (8) for $\nu>0$ and $z^{0}(t) \in(S-T) \cap \Omega$ for all $t$.

By the same way as the proof of Proposition 3.2, one can see that $u(z)$ is analytically continued from $\widehat{z}$ to itself along $\gamma^{0} \subset(S-T) \cap \Omega$. The obtained function element is holomorphic in a neighborhood $\omega$ of $\widehat{z}$.

From the assumption, $u(z)$ is also analytically continued along $\gamma^{\nu}$ for all $\nu$ except the end point $\widehat{z}$. Since $z^{\nu}(t)$ is continuous in $\{0 \leq \nu \leq 1,0 \leq t \leq 1\}$, the analytic continuation along $\gamma^{\nu}$ agrees with that along $\gamma^{0}$ in $\omega \backslash\{\vec{z}\}$. Hence we see $u(z)$ is continued analytically up to the end point $\widehat{z}$ along any $\gamma^{\nu}$ and therefore along $\gamma^{1}$.

It means $u(z)$ can be continued analytically along $\widetilde{\gamma}$ up to $\widehat{z}$, namely $\widehat{z}$ is a point of strong analytic continuation. By the same way as the proof of Proposition 3.4, one can prove $u(z)$ has a holomorphic extension in $\mathscr{R}(\Omega-K)$.

Remark 3.7. There is a topological difference between Propositions 3.5 and 3.6. We give an example to explain the necessity of assuming strong analytic continuation in Proposition 3.5. Let $k \in\{1,2, \cdots\}, \Omega:=\left\{\left|z_{i}\right|<1, i=1, \cdots, n\right\}, K^{1}:=$ $\left\{z_{n}=0\right\}$ and $K^{2}:=\left\{z_{n}=z_{1}^{k}\right\}$. If $k \geq 2$, the function $\log \left(z_{1}-z_{n}^{1 / k}\right)$ is holomorphic in the universal covering space $\mathscr{R}\left(\Omega-K^{1} \cup K^{2}\right)$. It can be analytically continued up to $\hat{z} \in\left(K^{2}-T\right)$ along some path but can not along some other path. When $k=1$, however, there are no such functions, because Proposition 3.6 applies with $S=$ $K^{1}$ and $K=K^{2}$ by exchanging $z_{1}$ with $z_{n}$. 
Proposition 3.8. Denote $V=\Omega-S \cup K^{1} \cup K^{2}$. Let $u(z)$ be holomorphic at a point $z^{\circ} \in V$ and have a holomorphic extension in $R(V)$. Then, if $u(z)$ has a point of weak analytic continuation $\widehat{z}$ on $T$, it has a unique holomorphic extension in $\Omega$.

Proof. $u(z)$ is holomorphic in a neighborhood $\omega$ of $\widehat{z} \in T$ and it is easy to see that any closed path in $V$ is homotope with a closed path in $\omega \cap V$. Hence $u(z)$ must be single valued and one can prove this proposition in the same way as the proof of Proposition 3.2.

(Q.E.D.)

\section{\$4. Proof of Main Theorem}

In this section we prove the Main theorem. Hence Condition $\mathrm{N}$ and the indicial condition (3) are supposed.

\subsection{Proposition 4.1}

Let $K$ be one of $K^{i}$ s. Then the following proposition holds.

Proposition 4.1. Suppose the Condition $N$ and the indicial condition (3). Let $u(z)$ be holomorphic in a neighborhood of $z^{\circ} \in(\Omega-K)$, satisfy the equation $P u=$ 0 there and have a holomorphic extension in the universal covering $\mathscr{R}(\Omega-K)$. Then the origin $z=0 \in K$ is a point of weak analytic continuation of $u(z)$.

\subsection{Change of Variables}

From now on we will prove Proposition 4.1. For that purpose we will make change of variables of the following form several times.

$$
w_{1}=z_{1}, \quad w_{j}=f_{j}(z), \quad j=2, \cdots, n
$$

with $f_{j}\left(0, z^{\prime}\right)=z_{j}$. Correspondingly $\zeta$ is transformed as

$$
\zeta_{1}=\eta_{1}+\sum_{k}^{\prime}\left(\partial w_{k} / \partial z_{1}\right) \eta_{k}, \quad \zeta_{j}=\sum_{k}^{\prime}\left(\partial w_{k} / \partial z_{j}\right) \eta_{k} \quad \text { for } j \geq 2
$$

It is clear that $\left\{z_{1}=0\right\},\left\{z_{1}=z_{n}=0\right\}$ and $\left\{z_{1}=0, \zeta^{\prime}=\nu^{\prime}\right\}$ correspond $\left\{w_{1}=0\right\}$, $\left\{w_{1}=w_{n}=0\right\}$ and $\left\{w_{1}=0, \eta^{\prime}=\nu^{\prime}\right\}$ respectively.

Set $\mu^{i}=-\sum_{k}^{\prime}\left(\partial w_{k} / \partial z_{1}\right) \eta_{k}+\lambda^{i}$. Then we easily see

$$
\begin{aligned}
P_{2}=a\left\{\zeta_{1}-\lambda^{1}\right\}\left\{\zeta_{1}-\lambda^{2}\right\} & =a\left\{\eta_{1}-\mu^{1}\right\}\left\{\eta_{1}-\mu^{2}\right\} \\
\left.\frac{\partial P_{2}}{\partial \eta_{1}}\right|_{\eta_{1}=\mu^{1}} & =\left.\frac{\partial P_{2}}{\partial \zeta_{1}}\right|_{\zeta_{1}=\lambda^{1}} \\
\left\{\eta_{1}-\mu^{1}, a\left\{\eta_{1}-\mu^{2}\right\}\right\} & =\left\{\zeta_{1}-\lambda^{1}, a\left\{\zeta_{1}-\lambda^{2}\right\}\right\}
\end{aligned}
$$

Condition $\mathrm{N}$ is thus invariant, and hence so are $A^{i}$ and $B^{i}$ and the indicial 
polynomials $F^{i}(\mu)$.

\subsection{Reduction to Three Standard Forms}

Now, write the operators $P$ in the form

$$
P=a(z)\left\{\left(D_{1}+\dot{\sum}_{j} a_{1 j} D_{j}\right)^{2}-\dot{\sum}_{j, k}^{\prime} a_{j k} D_{j} D_{k}\right\}+b_{1} D_{1}+\sum_{j}^{\prime} b_{j} D_{j}+c
$$

where $\sum_{j}^{\prime}$ and $\sum_{j k}^{\prime}$ denote the sum for $j \geq 2$ and for $j, k \geq 2$ respectively. It easily follows from 1) of Condition $\mathrm{N}$ that all the coefficients are holomorphic in a neighborhood of the origin. (Note $a(0) \neq 0$ is not assumed.)

Define $f_{i}(z)$ by

$$
D_{1} f+\sum_{j}^{\prime} a_{1 j} D_{j} f=0, \quad f\left(0, z^{\prime}\right)=z_{i}
$$

$i=2, \cdots, n$, and change the variables by

$$
w_{1}=z_{1}, \quad w_{i}=f_{i}(z), \quad i=2, \cdots, n
$$

Denote $w$ by $z$ again. Then the transformed operator, denoted by $P$ again, has the following form.

$$
P=a(z)\left\{D_{1}^{2}-\sum_{j, k}^{\prime} a_{j k} D_{j} D_{k}\right\}+b_{1} D_{1}+\sum_{j}^{\prime} b_{j} D_{j}+c
$$

where $a, a_{j k}, b_{1}, b_{j}$ and $c$ are all holomorphic in a neighborhood of the origin. Besides, Condition $N$, the phase functions $\varphi^{i}$ and the indicial polynomials $F^{i}(\mu)$ are all invariant under this change of variables.

Taking a branch of $\sqrt{ }$ appropriately, we have

$$
\lambda^{1}=\delta, \quad \lambda^{2}=-\delta, \quad \delta=\sqrt{\sum_{j, k}^{\prime} a_{j k} \zeta_{j} \zeta_{k}},
$$

which are, by the assumption, holomorphic in a neighborhood of $\left(z, \zeta^{\prime}\right)=\left(0, \nu^{\prime}\right)$.

It follows from 2) of Condition $\mathrm{N}$ that

$$
Q^{1}=2 a(z) \delta\left(z, \zeta^{\prime}\right)=0 \text { on }\left\{z_{1}=0, \zeta^{\prime}=\nu^{\prime}\right\}
$$

Since $a$ and $\delta$ are holomorphic, we have

$$
a\left(0, z^{\prime}\right) \equiv 0 \quad \text { or } \quad \delta\left(0, z^{\prime}, \nu^{\prime}\right) \equiv 0 .
$$

Next, note that

$$
\left\{\zeta_{1}-\delta\left(z, \zeta^{\prime}\right), a(z)\left\{\zeta_{1}+\delta\left(z, \zeta^{\prime}\right)\right\}\right\}
$$




$$
=\left\{\zeta_{1}-\delta, a\right\}\left(\zeta_{1}-\delta\right)+2\left\{\zeta_{1}, a \delta\right\}-2\{\delta, a\} \delta
$$

Then we have

$$
A^{1}=\left.2 \frac{\partial(a \delta)}{\partial z_{1}}\right|_{\left(z, \zeta^{\prime}\right)=\left(0, \nu^{\prime}\right)} \neq 0
$$

Hence the following two cases are possibe.

1st case: $a\left(0, z^{\prime}\right) \equiv 0, \quad D_{1} a(0) \neq 0, \quad \delta\left(0, \nu^{\prime}\right) \neq 0$

2nd case: $a(0) \neq 0, \quad \delta\left(0, z^{\prime}, \nu^{\prime}\right) \equiv 0, \quad\left(\partial \delta / \partial z_{1}\right)\left(0, \nu^{\prime}\right) \neq 0$

In the 1 st case, one can write $a=z_{1} \tilde{a}, \tilde{a}(0) \neq 0$, and, by omitting $\sim, P$ is written as

$$
P=z_{1} a\left\{D_{1}^{2}-\sum_{j, k}^{\prime} a_{j k} D_{j} D_{k}\right\}+b_{1} D_{1}+\sum_{j}^{\prime} b_{j} D_{j}+c
$$

where $a(0) \neq 0, a_{n n}(0) \neq 0$.

In the 2 nd case, one can write $a_{n n}=z_{1}^{2} \widetilde{a}_{n n}, \widetilde{a}_{n n}(0) \neq 0$. Omit $\sim$ and set

$$
\Delta\left(z, \zeta^{\prime}\right):=a_{n n} z_{1}^{2} \zeta_{n}^{2}+2 \sum_{j}^{\prime \prime} a_{j n} \zeta_{j} \zeta_{n}+\sum_{j, k}^{\prime \prime} a_{j k} \zeta_{j} \zeta_{k}
$$

where $\sum_{j}^{\prime \prime}$ and $\sum_{j, k}^{\prime \prime}$ denote the sum for $2 \leq_{j} \leq n-1$ and for $2 \leq_{j, k} \leq n-1$ respectively.

By Condition N. $\sqrt{\Delta\left(z, \zeta^{\prime}\right)}$ is holomorphic in a conic neighborhood of $(z$, $\left.\zeta^{\prime}\right)=\left(0, \nu^{\prime}\right)$, say $V_{\varepsilon}:=\left\{\left(z, \zeta^{\prime}\right) ;\left|z_{i}\right|<\varepsilon \forall i \leq n,\left|\zeta_{i} / \zeta_{n}\right|<\varepsilon \forall i \leq n-1\right\}$. Therefore $\Delta$ can not have simple zeros there.

Suppose $a_{j n}\left(0, z^{\prime}\right) \not \equiv 0$ for some $2 \leq j \leq n-1$. Fix $z^{\prime}$ near the origin and let $z_{1}$ $=0, \zeta_{k}=0$ for $k \neq j$. Then

$$
\Delta=2 a_{j n}\left(0, z^{\prime}\right) \zeta_{j} \zeta_{n}+a_{j j}\left(0, z^{\prime}\right) \zeta_{j}^{2},
$$

which vanishes on $\zeta_{j}=0$ with degree 1 . It is a contradiction, and hence we see $a_{j n}\left(0, z^{\prime}\right) \equiv 0$ for all $2 \leq j \leq n-1$.

Denote $a_{j n}=z_{1} \widetilde{a_{j n}}$, omit $\sim$ and write

$$
\begin{aligned}
\Delta\left(z, \zeta^{\prime}\right) & =a_{n n} z_{1}^{2} \zeta_{n}^{2}+2 z_{1} \sum_{j}^{\prime \prime} a_{j n} \zeta_{j} \zeta_{n}+\sum_{j, k}^{\prime \prime} a_{j k} \zeta_{j} \zeta_{k} \\
& =a_{n n}\left\{\left(z_{1} \zeta_{n}+\alpha\left(z, \zeta^{\prime \prime}\right)\right)^{2}-\beta\left(z, \zeta^{\prime \prime}\right)\right\}
\end{aligned}
$$

where $\alpha\left(z, \zeta^{\prime \prime}\right)=\sum_{j}^{\prime \prime}\left(a_{j n} / a_{n n}\right) \zeta_{j}$ and $\beta\left(z, \zeta^{\prime \prime}\right)=-\sum_{j, k}^{\prime \prime}\left(a_{j k} / a_{n n}\right) \zeta_{j} \zeta_{k}+\alpha^{2}\left(z, \zeta^{\prime \prime}\right)$.

Put $z^{\prime}=\widehat{z}^{\prime}, \zeta_{n}=1, \zeta^{\prime \prime}=\tau \widehat{\zeta}^{\prime \prime}, \tau \in \mathbb{C}$, then

$$
\Delta=a_{n n}\left\{\left(z_{1}+\tau \alpha\left(z_{1}, \widehat{z}^{\prime}, \hat{\zeta}^{\prime \prime}\right)\right)^{2}-\tau^{2} \beta\left(z_{1}, \widehat{z}^{\prime}, \widehat{\zeta}^{\prime \prime}\right)\right\} .
$$


It vanishes on

$$
z_{1}+\tau\left\{\alpha\left(z_{1}, \widehat{z}^{\prime}, \widehat{\zeta}^{\prime \prime}\right) \pm \sqrt{\beta\left(z_{1}, \widehat{z}^{\prime}, \widehat{\zeta}^{\prime \prime}\right)}\right\}=0
$$

Suppose $\alpha\left(0, z^{\prime}, \zeta^{\prime \prime}\right) \not \equiv 0$. Suppose also $\beta\left(z_{1}, z^{\prime}, \zeta^{\prime \prime}\right) \not \equiv 0$. Since $\alpha, \beta$ are holomorphic, there are $\left(\widehat{z}^{\prime}, \widehat{\zeta}^{\prime \prime}\right)$ such that $\alpha\left(0, \widehat{z}^{\prime}, \widehat{\zeta}^{\prime \prime}\right) \neq 0$ and $\beta\left(z_{1}, \widehat{z}^{\prime}, \widehat{\zeta}^{\prime \prime}\right) \not \equiv 0$ as a function of $z_{1}$. Therefore there is a $z_{1}=\widehat{z_{1}}$ with $0<\left|\widehat{z_{1}}\right|<\varepsilon$ such that $\Delta$ has at least one simple zero $\tau$ with $|\tau|<1$. Therefore, if $\alpha\left(0, z^{\prime}, \zeta^{\prime \prime}\right) \not \equiv 0$, then $\beta\left(z_{1}, z^{\prime}\right.$, $\left.\zeta^{\prime \prime}\right)$ must vanish identically and so the operator $P$ can be written in the form

$$
P=a\left\{D_{1}^{2}-\left\{\sum_{j}^{\prime \prime} a_{j} D_{j}+z_{1} a_{n} D_{n}\right\}^{2}\right\}+b_{1} D_{1}+\sum_{j}^{\prime} b_{j} D_{j}+c
$$

where the coefficients are all holomorphic in a neighborhood of $z=0, a(0) \neq 0$ and $a_{n}(0) \neq 0$.

Next, suppose $\alpha\left(0, z^{\prime}, \zeta^{\prime \prime}\right) \equiv 0$. If $\beta\left(0, z^{\prime}, \zeta^{\prime \prime}\right) \not \equiv 0$, by the same reason as above, $\Delta$ has simple zeros in $V_{\varepsilon}$. Else if $\beta\left(0, z^{\prime}, \zeta^{\prime \prime}\right) \equiv 0$ and $\left(\partial \beta / \partial z_{1}\right)\left(0, z^{\prime}, \zeta^{\prime \prime}\right)$ $\not \equiv 0$, then

$$
\Delta=\left(\partial \beta / \partial z_{1}\right)\left(0, z^{\prime}, \zeta^{\prime \prime}\right) z_{1}+O\left(z_{1}^{2}\right)
$$

which vanishes on $z_{1}=0$ with degree 1 at some $\left(z^{\prime}, \zeta^{\prime \prime}\right)$. Thus we see, if $\alpha\left(z_{1}, z^{\prime}\right.$, $\left.\zeta^{\prime \prime}\right)=O\left(z_{1}\right)$, then $\beta\left(z_{1}, z^{\prime}, \zeta^{\prime \prime}\right)=O\left(z_{1}^{2}\right)$. It follows from this that

$$
a_{j n}=O\left(z_{1}\right) \text { for } 2 \leq j \leq n-1, a_{j k}=O\left(z_{1}^{2}\right) \text { for } 2 \leq_{j, k} \leq n-1
$$

as $z_{1} \rightarrow 0$. Denoting $a_{j n}=z_{1} \widetilde{a_{j n}}, a_{j k}=z_{1}^{2} \widetilde{a_{j k}}$, and omitting $\sim$, one can write

$$
P=a\left\{D_{1}^{2}-z_{1}^{2} \sum_{j, k}^{\prime} a_{j k} D_{j} D_{k}\right\}+b_{1} D_{1}+\sum_{j}^{\prime} b_{j} D_{j}+c
$$

where the coefficients are all holomorphic in a neighborhood of $z=0, a(0) \neq 0$ and $a_{n n}(0) \neq 0$.

Thus the following proposition has been proved.

Proposition 4.2. Every operator satisfying Condition $N$ is reduced to one of the following three operators.

$$
\begin{aligned}
& P^{A}=a\left\{D_{1}^{2}-\left\{\sum_{j}^{\prime \prime} a_{j} D_{j}+z_{1} a_{n} D_{n}\right\}^{2}\right\}+b_{1} D_{1}+\sum_{j}^{\prime} b_{j} D_{j}+c \\
& P^{B}=a\left\{D_{1}^{2}-z_{1}^{2} \sum_{j, k}^{\prime} a_{j k} D_{j} D_{k}\right\}+b_{1} D_{1}+\sum_{j}^{\prime} b_{j} D_{j}+c
\end{aligned}
$$




$$
P^{C}=z_{1} a\left\{D_{1}^{2}-\sum_{j, k}^{\prime} a_{j k} D_{j} D_{k}\right\}+b_{1} D_{1}+\sum_{j}^{\prime} b_{j} D_{j}+c
$$

where the coefficients are all holomorphic in a neighborhood of the origin, $\sum_{j}^{\prime \prime}$ denotes the sum for $2 \leq j \leq n-1, a(0) \neq 0, a_{n}(0) \neq 0$ and $a_{n n}(0) \neq 0$.

\subsection{Cauchy-Kovalevskaya Type Theorem}

Recall the Cauchy Kovalevskaya type theorem obtained in our preceding paper [8].

Definition 4.3. Let $m, r$ be integers with $0 \leq r \leq m$ and $\sigma$ be a real number with $0 \leq \sigma \leq 1 . \mathscr{L}^{m, r, \sigma}$ denotes the set of operators with the form

$$
L:=\sum_{s=0}^{r} a_{s}(z)\left(z_{1} D_{1}\right)^{r-s} D_{n}^{m-r}+\sum_{s=0}^{m} \sum_{\alpha \in A(m-s)} b_{\alpha}(z) z_{1}^{l(\alpha)} D^{\alpha}
$$

where $A(m-s)=\left\{\alpha ;|\alpha|=m-s, \alpha_{n} \leq m-r, \alpha \neq(r-s, 0, \cdots, 0, m-r)\right\}$, the coefficents are all holomorphic in a neighborhood $\Omega$ of the origin $z=0$ and each $l(\alpha)$ is a non-negative integer satisfying

$$
\sigma l(\alpha)+(1-\sigma) \alpha_{1} \geq_{r}-s
$$

Setting

$$
I_{L}(\lambda):=\sum_{s=0}^{r} a_{s}(0) \lambda^{r-s},
$$

we call it the indicial polynomial of $L$ with respect to $z_{n}=0$.

Note the hyperplane $z_{n}=h, h \in \mathbb{C}$, is characteristic for $L$ with multiplicity at least $r$. Let $U=\left\{z ;\left|z_{j}\right|<\rho\right\} \subset \Omega$ and consider the characteristic Cauchy problem

$$
L v=0, v-w=O\left\{\left(z_{n}-h\right)^{m-r}\right\} \text { as } z_{n} \rightarrow h
$$

Theorem 4.4 [8, Th.1]. Suppose $a_{0}(0) \neq 0$ and $I_{L}(\lambda) \neq 0, \forall \lambda \in\{0,1,2, \cdots\}$. Then there are two positive constants $\kappa, \rho^{\prime}(<\rho)$ such that for any $h \in \mathbb{C}$ with $|h|<\kappa$ and any $w$ holomorphic in $U$ there exists a unique holomorphic solution $v(z)$ to the Cauchy problem (15) in $U_{h}^{\prime}=\left\{z ;\left|z_{j}\right|<\rho^{\prime}\right.$ for $\left.j<n,\left|z_{n}-h\right|<\rho^{\prime}\right\}$.

\subsection{Proof of Proposition 4.1}

To apply the above theorem to the operators satisfying Condition $\mathrm{N}$, we need to change the variables. We will consider only the case $K=K^{1}$. 
First, consider the case $P=P^{A}$. Define $f_{j}(z)$ by

$$
D_{1} f-\lambda^{1}\left(z, D^{\prime} f\right)=0, f\left(0, z^{\prime}\right)=z_{j}
$$

$j=2, \cdots, n$, where $\lambda^{1}\left(z, \zeta^{\prime}\right)=\sum_{j}^{\prime \prime} a_{j} \zeta_{j}+z_{1} a_{n} \zeta_{n}$.

Change the variables by

$$
w_{1}=z_{1}, w_{j}=f_{j}(z), j=2, \cdots, n .
$$

Denote $w$ by $z$ again and the transformed operator by $\widetilde{P}^{A}$. As we have remarked before, the Condition $\mathrm{N}$, phase functions $\varphi^{i}$ and the indicial polynomials $F^{\imath}(\mu)$ are invariant under this change of variables.

Besides, $\widetilde{P}^{A}$ has the following form.

$$
\widetilde{P}^{A}=a(z)\left\{D_{1}+\sum_{j}^{\prime \prime} a_{j}(z) D_{j}+z_{1} a_{n} D_{n}\right\} D_{1}+b_{1} D_{1}+\sum_{j}^{\prime} b_{j} D_{j}+c
$$

where $a(0) \neq 0$ and $a_{n}(0) \neq 0$. It is easy to see that the transformed operator belongs to $\mathscr{L}^{2,1, \sigma}$ with $\sigma=0$.

Since $\varphi^{1}$ is the solution of $D_{1} \varphi=0, \varphi\left(0, z^{\prime}\right)=z_{n}$, we have $\varphi^{1}=z_{n}$ and $K^{1}=$ $\left\{z_{n}=0\right\}$. Besides,

$$
A^{1}=\left\{\zeta_{1}, a(z)\left\{\zeta_{1}+\sum_{j}^{\prime \prime} a_{j}(z) \zeta_{j}+z_{1} a_{n} \zeta_{n}\right\}\right\}_{\zeta_{1}=0,\left(z, \zeta^{\prime}\right)=\left(0, \nu^{\prime}\right)}=a(0) a_{n}(0)
$$

and $B^{1}=\widetilde{P}^{A} z_{n}=b_{n}(0)$. So we have $I_{\widetilde{P}^{A}}(\mu)=F^{1}(\mu)$. Therefore, if we take $\rho>0$ so small, Theorem 4.4 applies to $\widetilde{P}^{A}$ in $U_{\rho}=\left\{z ;\left|z_{j}\right|<\rho\right\}$.

Let $0<h<\min \left\{\kappa, \rho^{\prime}\right\}$ and put $z^{h}=(0, \cdots, 0, h)$. Continue $u(z)$ along a path in $\Omega-K^{1}$ up to $z^{h}$. Its function element at $z^{h}$ determines a single valued holomorphic function in $U_{\rho} \cap\left\{\operatorname{Re} z_{n}>0\right\}$.

Consider the characteristic Cauchy problem

$$
\widetilde{P}^{A} v=0, v-w=O\left(z_{n}-h\right) \text { as } z_{n} \rightarrow h
$$

with $w=u\left(z_{1}, \cdots, z_{n-1}, h\right)$. By the Theorem 4.4 , there exists a unique holomorphic solution $v(z)$ in $U_{h}^{\prime}=\left\{z ;\left|z_{j}\right|<\rho^{\prime} \forall j \leq n-1,\left|z_{n}-h\right|<\rho^{\prime}\right\}$. Since $v=u$ in a neighborhood of $z^{h}$ and $0 \in U_{h}^{\prime}, v$ is a holomorphic extension of $u$ to a neighborhood of the origin. Thus Proposition 4.1 has been proved in the case of $P=P^{A}$.

Next, consider the case $P=P^{B}$. Set $\delta\left(z, \zeta^{\prime}\right):=\sqrt{\sum_{j, k}^{\prime} a_{j k} \zeta_{j} \zeta_{k}}, \lambda^{1}:=z_{1} \delta$ and $\lambda^{2}:=-z_{1} \delta$ in a neighborhood of $\left(z, \zeta^{\prime}\right)=\left(0, \nu^{\prime}\right)$.

Recall $\varphi^{1}$ is the solution of

$$
D_{1} \varphi-\lambda^{1}\left(z, D^{\prime} \varphi\right)=0, \varphi\left(0, z^{\prime}\right)=z_{n}
$$


and change the variables by

$$
w_{j}=z_{j}, j=1, \cdots, n-1, w_{n}=\varphi^{1}(z)
$$

The Condition $\mathrm{N}$, phase functions $\varphi^{i}$ and the indicial polynomials $F^{i}(\mu)$ are invariant under this change of variables.

Denote $w$ by $z$ again and the transformed operator by $\widetilde{P}^{B}$. Then the principal symbol is decomposed as

$$
\widetilde{P}_{2}^{B}(z, \zeta)=a\left\{\zeta_{1}-z_{1} \mu^{1}\left(z, \zeta^{\prime}\right)\right\}\left\{\zeta_{1}-z_{1} \mu^{2}\left(z, \zeta^{\prime}\right)\right\}
$$

where $\mu^{i}\left(z, \zeta^{\prime}\right)$ are holomorphic near $\left(0, \nu^{\prime}\right)$ and $\mu^{1}\left(z, \nu^{\prime}\right) \equiv 0$.

Note $\varphi^{1}=z_{n}$ and $K^{1}=\left\{z_{n}=0\right\}$. It is easy to see that $\widetilde{P}^{B}$ has the following form.

$$
\widetilde{P}^{B}=a\left\{D_{1}^{2}+z_{1} a_{1 n} D_{1} D_{n}+z_{1}^{2} \sum_{j, k}^{\prime} a_{j k} D_{j} D_{k}\right\}+b_{1} D_{1}+\sum_{j}^{\prime} b_{j} D_{j}+c
$$

where the coefficients are all holomorphic, $a_{1 n}(0) \neq 0$ and $a_{n n}(z) \equiv 0$. Therefore $\widetilde{P}^{B}$ belongs to $\mathscr{L}^{2,1, \sigma}$ with $\sigma=1 / 2$.

By the way, one can see

$$
\begin{aligned}
A^{1} & =\left\{\zeta_{1}-z_{1} \mu^{1}\left(z, \zeta^{\prime}\right), a(z)\left(\zeta_{1}-z_{1} \mu^{2}\left(z, \zeta^{\prime}\right)\right)\right\}_{\zeta_{1}=z_{1} \mu^{1}\left(z, \zeta^{\prime}\right),\left(z, \zeta^{\prime}\right)=\left(0, \nu^{\prime}\right)} \\
& =a(0)\left\{\mu^{1}\left(0, \nu^{\prime}\right)-\mu^{2}\left(0, \nu^{\prime}\right)\right\}
\end{aligned}
$$

Since $\mu^{1}\left(0, \nu^{\prime}\right)=0$, we have $A^{1}=a(0) a_{1 n}(0)$. Clearly, $B^{1}=\widetilde{P}^{B} z_{n}=b_{n}(0)$. Hence we have $I_{\widetilde{P}^{B}}(\mu)=F^{1}(\mu)$. Therefore Theorem 4.4 applies to the operator $\widetilde{P}^{B}$, and one can prove Proposition 4.1 in the same way as in the case $P=P^{A}$.

Lastly, consider the case $P=P^{C}$. Set $\lambda^{1}\left(z, \zeta^{\prime}\right):=\sqrt{\sum_{j, k}^{\prime} a_{j k} \zeta_{j} \zeta_{k}}$ and $\lambda^{2}:=-\lambda^{1}$ in a neighborhood of $\left(z, \zeta^{\prime}\right)=\left(0, \nu^{\prime}\right)$.

Recall $\varphi^{1}$ is the solution of

$$
D_{1} \varphi-\lambda^{1}\left(z, D^{\prime} \varphi\right)=0, \quad \varphi\left(0, z^{\prime}\right)=z_{n}
$$

and change the variables by

$$
w_{j}=z_{j}, j=1, \cdots, n-1, \quad w_{n}=\varphi^{1}(z)
$$

The Condition $N$, phase functions $\varphi^{i}$ and the indicial polynomials $F^{i}(\mu)$ are invariant under this change of variables.

Denote $w$ by $z$ again and the transformed operator by $\tilde{P}^{c}$. Then the principal symbol is decomposed as

$$
\widetilde{P}_{2}^{C}(z, \zeta)=a z_{1}\left\{\zeta_{1}-\mu^{1}\left(z, \zeta^{\prime}\right)\right\}\left\{\zeta_{1}-\mu^{2}\left(z, \zeta^{\prime}\right)\right\}
$$

where $\mu^{i}\left(z, \zeta^{\prime}\right)$ are holomorphic near $\left(0, \nu^{\prime}\right)$ and $\mu^{1}\left(z, \nu^{\prime}\right) \equiv 0$. 
Note $\varphi^{1}=z_{n}$ and $K^{1}=\left\{z_{n}=0\right\}$. It is easy to see that $\widetilde{P}^{c}$ has the following form.

$$
\widetilde{P}^{C}=a z_{1}\left\{D_{1}^{2}+a_{1 n} D_{1} D_{n}+\dot{\sum}_{j, k} a_{j k} D_{j} D_{k}\right\}+b_{1} D_{1}+\sum_{j}^{\prime} b_{j} D_{j}+c
$$

where the coefficients are all holomorphic, $a_{1 n}(0) \neq 0$ and $a_{n n}(z) \equiv 0$. Therefore $\widetilde{P}^{C}$ belongs to $\mathscr{L}^{2,1, \sigma}$ with $\sigma=1$.

By the way, one can see

$$
\begin{aligned}
A^{1} & =\left\{\zeta_{1}-\mu^{1}\left(z, \zeta^{\prime}\right), a(z) z_{1}\left\{\zeta_{1}-\mu^{2}\left(z, \zeta^{\prime}\right)\right\}\right\}_{\zeta_{1}=\mu^{1}\left(z, \zeta^{\prime}\right),\left(z, \zeta^{\prime}\right)=\left(0, \nu^{\prime}\right)} \\
& =a(0)\left\{\mu^{1}\left(0, \nu^{\prime}\right)-\mu^{2}\left(0, \nu^{\prime}\right)\right\} .
\end{aligned}
$$

Since $\mu^{1}\left(0, \nu^{\prime}\right)=0$, we have $A^{1}=a(0) a_{1 n}(0)$. Clearly, $B^{1}=\widetilde{P}^{c} z_{n}=b_{n}(0)$. Hence we have $I_{\widetilde{P}^{c}}(\mu)=F^{1}(\mu)$. Therefore Theorem 4.4 applies to the operator $\widetilde{P}^{C}$, and one can prove Proposition 4.1 in the same way as in the case $P=P^{A}$.

We have thus finished the proof of Proposition 4.1.

\subsection{End of the Proof}

By the assumption, $u(z)$ is holomorphic at $z^{\circ} \in V=\Omega-S \cup K^{1} \cup K^{2}$, satisfies the equation $P u=0$, has a holomorphic extension in the universal covering $\mathscr{R}(V)$, and has a point of strong analytic continuation $\widehat{z}$ on $K^{1} \cup K^{2}$ and a point of weak one $a \in(S-T) \Omega$.

If $\widehat{z} \in T, u(z)$ has a holomorphic extension in $\Omega$ by Proposition 3.8. If $\widehat{z} \in$ $\left(K^{2}-T\right.$ ) (or $\in\left(K^{1}-T\right)$ ), it follows from Proposition 3.4 that $u(z)$ has a holomorphic extension in the universal covering $\mathscr{R}\left(\Omega-S \cup K^{1}\right)$ (or in $\mathscr{R}(\Omega-S \cup$ $K^{2}$ ) respectively).

By Proposition 3.6, $u(z)$ has a holomorphic extension in $\Omega-K$. ( $K$ is one of $K^{v}$ s.) Next, by Proposition $4.1, z=0$ is a point of weak analytic continuation. Lastly by Proposition $3.3, u(z)$ has a holomorphic extension in $\Omega$. Thus we have finished the proof of the Main theorem.

\subsection{Remarks}

A. Concrete expression of the indicial equation. We give a concrete expression of the indicial equation in terms of the coefficients of $P^{A}, P^{B}$ and $P^{C}$.

In the case $P=P^{A}$, set $\lambda^{ \pm}= \pm\left(\sum_{j}^{\prime \prime} a_{j} \zeta_{j}+z_{1} a_{n} \zeta_{n}\right)$. Let $\varphi^{ \pm}$be the solution of

$$
D_{1} \varphi-\lambda^{ \pm}\left(z, D^{\prime} \varphi\right)=0, \varphi\left(0, z^{\prime}\right)=z_{n}
$$

where the sign + and - should be taken in its order. $K^{ \pm}:=\left\{z ; \varphi^{ \pm}(z)=0\right\}$ are characteristic surfaces issued from $T$.

By the definition 


$$
\begin{aligned}
A^{+} & =\left\{\zeta_{1}-\lambda^{+}, a\left\{\zeta_{1}-\lambda^{-}\right\}\right\}_{\zeta_{1}=\lambda^{+},\left(z, \zeta^{\prime}\right)=\left(0, \nu^{\prime}\right)} \\
& =\left\{\zeta_{1}-\lambda^{+}, 2 a \lambda^{+}\right\}_{\zeta_{1}=\lambda^{+},\left(z, \zeta^{\prime}\right)=\left(0, \nu^{\prime}\right)}=2 a(0) a_{n}(0) \\
B^{+} & =P \varphi^{+}(0)=a(0) D_{1}^{2} \varphi^{+}(0)+b_{1}(0) D_{1} \varphi^{+}(0)+b_{n}(0) D_{n} \varphi^{+}(0) \\
& =a(0) a_{n}(0)+b_{n}(0)
\end{aligned}
$$

In the same way

$$
A^{-}=-2 a(0) a_{n}(0), B^{-}=-a(0) a_{n}(0)+b_{n}(0)
$$

Hence the indicial polynomial of $P^{A}$ corresponding to $K^{ \pm}$is respectively

$$
F_{A}^{ \pm}(\mu)= \pm a(0) a_{n}(0)\{2 \mu+1\}+b_{n}(0)
$$

In the case $P=P^{B}$, set $\delta:=\sqrt{\sum_{j, k}^{\prime} a_{j k}(z) \zeta_{j} \zeta_{k}}$ in a neighborhood of $\left(z, \zeta^{\prime}\right)=$ $\left(0, \nu^{\prime}\right)$ and $\lambda^{ \pm}= \pm z_{1} \delta\left(z, \zeta^{\prime}\right)$. Define $\varphi^{ \pm}$and $K^{ \pm}$in the same way as in the case $P$ $=P^{A}$.

By the definition

$$
\begin{aligned}
A^{+} & =\left\{\zeta_{1}-z_{1} \delta, a\left\{\zeta_{1}+z_{1} \delta\right\}\right\}_{\zeta_{1}=z_{1} \delta,\left(z, \zeta^{\prime}\right)=\left(0, \nu^{\prime}\right)} \\
& =\left\{\zeta_{1}-z_{1} \delta, 2 a z_{1} \delta\right\}_{\zeta_{1}=z_{1} \delta,\left(z, \zeta^{\prime}\right)=\left(0, \nu^{\prime}\right)}=2 a(0) \sqrt{a_{n n}(0)} \\
B^{+} & =P \varphi^{+}(0)=a(0) D_{1}^{2} \varphi^{+}(0)+b_{1}(0) D_{1} \varphi^{+}(0)+b_{n}(0) D_{n} \varphi^{+}(0) \\
& =a(0) \sqrt{a_{n n}(0)}+b_{n}(0)
\end{aligned}
$$

In the same way

$$
A^{-}=-2 a(0) \sqrt{a_{n n}(0)}, B^{-}=-a(0) \sqrt{a_{n n}(0)}+b_{n}(0)
$$

Hence the indicial polynomial of $P^{B}$ corresponding to $K^{ \pm}$is respectively

$$
F_{B}^{ \pm}(\mu)= \pm a(0) \sqrt{a_{n n}(0)}\{2 \mu+1\}+b_{n}(0)
$$

In the case $P=P^{C}$, set $\delta:=\sqrt{\sum_{j, k}^{\prime} a_{j k}(z) \zeta_{j} \zeta_{k}}$ in a neighborhood of $\left(z, \zeta^{\prime}\right)=$ $\left(0, \nu^{\prime}\right)$ and $\lambda^{ \pm}= \pm \delta\left(z, \zeta^{\prime}\right)$. Define $\varphi^{ \pm}$and $K^{ \pm}$in the same way as in the case $P$ $=P^{A}$.

By the definition

$$
\begin{aligned}
A^{+} & =\left\{\zeta_{1}-\delta, a z_{1}\left\{\zeta_{1}+\delta\right\}\right\}_{\zeta_{1}=\delta,\left(z, \zeta^{\prime}\right)=\left(0, \nu^{\prime}\right)} \\
& =\left\{\zeta_{1}-\delta, 2 a z_{1} \delta\right\}_{\zeta_{1}=\delta,\left(z, \zeta^{\prime}\right)=\left(0, \nu^{\prime}\right)}=2 a(0) \sqrt{a_{n n}(0)} \\
B^{+} & =P \varphi^{+}(0)=b_{1}(0) D_{1} \varphi^{+}(0)+b_{n}(0) D_{n} \varphi^{+}(0) \\
& =b_{1}(0) \sqrt{a_{n n}(0)}+b_{n}(0)
\end{aligned}
$$

In the same way

$$
A^{-}=-2 a(0) \sqrt{a_{n n}(0)}, B^{-}=-b_{1}(0) \sqrt{a_{n n}(0)}+b_{n}(0)
$$

Hence the indicial polynomial of $P^{C}$ corresponding to $K^{ \pm}$is respectively

$$
F_{C}^{ \pm}(\mu)= \pm \sqrt{a_{n n}(0)}\left\{2 a(0) \mu+b_{1}(0)\right\}+b_{n}(0)
$$


B. On $K^{1} \cap K^{2}=T$. It is mentioned in $\S 2$ that one may suppose $K^{1} \cap K^{2}=T$ by taking $r$ appropriately. One can verify it by Proposition 4.2 easily, however it should be proved directly without using change of variables.

It follows from 2) of Condition $\mathrm{N}$ that

$$
a(z)\left\{\lambda^{1}\left(z, \zeta^{\prime}\right)-\lambda^{2}\left(z, \zeta^{\prime}\right)\right\}=0 \text { on }\left\{z_{1}=0, \zeta^{\prime}=\nu^{\prime}\right\} .
$$

Since $a$ and $\lambda^{i}$ s are holomorphic, we have two cases.

Case A: $\quad a=0 \quad$ on $z_{1}=0$

Case B: $\lambda^{1}-\lambda^{2}=0$ on $z_{1}=0, \zeta^{\prime}=\nu^{\prime}$

In the case $\mathrm{A}$, we have

$$
\left\{\zeta_{1}-\lambda^{1}, a \cdot\left(\zeta_{1}-\lambda^{2}\right)\right\}_{\zeta_{1}=\lambda^{1}}=D_{1} a \cdot\left(\lambda^{1}-\lambda^{2}\right) \text { on } z_{1}=0 .
$$

Hence, by 3 ) of Condition $\mathrm{N}$,

$$
D_{1} a(0) \neq 0, \lambda^{1}\left(0, \nu^{\prime}\right) \neq \lambda^{2}\left(0, \nu^{\prime}\right) .
$$

Therefore

$$
D_{1} \Phi^{1}(0)-D_{1} \Phi^{2}(0)=\lambda^{1}\left(0, \nu^{\prime}\right)-\lambda^{2}\left(0, \nu^{\prime}\right) \neq 0 .
$$

It means $K^{1} \cap K^{2}=T$ near the origin.

In the case $B$, we have

$$
\left\{\zeta_{1}-\lambda^{1}, a \cdot\left(\zeta_{1}-\lambda^{2}\right)\right\}_{\zeta_{1}=\lambda^{1}}=a\left\{\zeta_{1}-\lambda^{1}, \zeta_{1}-\lambda^{2}\right\}_{\zeta_{1}=\lambda^{1}} \text { on } z_{1}=0 .
$$

Hence, by 3) of Condition $\mathrm{N}$,

$$
a(0) \neq 0,\left\{\zeta_{1}-\lambda^{1}, \zeta_{1}-\lambda^{2}\right\}_{\zeta_{1}=\lambda^{1},\left(z, \zeta^{\prime}\right)=\left(0, \nu^{\prime}\right)} \neq 0 .
$$

It is easy to see that

$$
D_{1} \Phi^{1}-D_{1} \Phi^{2}=\lambda^{1}\left(z, D^{\prime} \Phi^{1}\right)-\lambda^{2}\left(z, D^{\prime} \Phi^{2}\right)=0 \text { on } z_{1}=0,
$$

and that

$$
\begin{aligned}
& D_{1}^{2} \Phi^{1}-D_{1}^{2} \Phi^{2}=D_{1} \lambda^{1}\left(z, D^{\prime} \Phi^{1}\right)-D_{1} \lambda^{2}\left(z, D^{\prime} \Phi^{2}\right) \\
& =\lambda_{z_{1}}^{1}-\lambda_{z_{1}}^{2}+\sum_{j}^{r} \lambda_{\zeta_{j}}^{1} D_{1} D_{j} \Phi^{1}-\sum_{j}^{\lambda_{\zeta_{j}}^{2} D_{1} D_{j} \Phi^{2}} \\
& =\lambda_{z_{1}}^{1}-\lambda_{z_{1}}^{2}+\sum_{j}^{\infty} \lambda_{\zeta_{j}}^{1} \lambda_{z_{j}}^{1}-\sum_{j}^{\infty} \lambda_{\zeta_{j}}^{2} \lambda_{z_{j}}^{2} \\
& =\left\{\zeta_{1}-\lambda^{1}, \zeta_{1}-\lambda^{2}\right\}_{\zeta_{1}=\lambda^{1}} \text { on } z_{1}=0, \zeta^{\prime}=\nu^{\prime} .
\end{aligned}
$$

Here we used $D_{j} D_{k} \Phi^{i}\left(0, z^{\prime}\right)=0$ for $j, k \geq 2$ and $\lambda_{z_{j}}^{1}\left(0, z^{\prime}, \nu^{\prime}\right)=\lambda_{z j}^{2}\left(0, z^{\prime}, \nu^{\prime}\right)$ for $j \geq$ 
2. We thus see $K^{1} \cap K^{2}=T$ near the origin in the case $\mathrm{B}$, too.

\section{§5. Extension to Higher Order Equations}

\subsection{Generalization of Main Theorem}

Let $P$ be a partial differential operator of second order satisfying Condition $\mathrm{N}$ and put

$$
L:=P^{r}+Q_{1} P^{r-1}+\cdots+Q_{r}
$$

where $Q_{k}=Q_{k}(z, D)$ are partial differential operators of order $k$ with holomorphic coefficients.

Let $\lambda^{i}, K^{i}$ and $F^{i}(\mu)$ be those introduced in $\S 2$ for the operator $P$ and set

$$
G^{i}(\mu):=\left\{F^{i}(\mu)\right\}^{r}+C_{1}^{i}\left\{F^{i}(\mu)\right\}^{r-1}+\cdots+C_{r}^{i}
$$

where $C_{k}^{i}=\left.Q_{k}^{\circ}(z, \zeta)\right|_{\zeta_{1}=\lambda^{z}\left(z, \zeta^{\prime}\right),\left(z, \zeta^{\prime}\right)=\left(0, \nu^{\prime}\right)}$ and $Q_{k}^{\circ}$ stands for the homogenuous part of order $k$ of $Q_{k}$.

\section{Theorem 5.1. Assume}

$$
G^{i}(\mu) \neq 0 \quad \forall \mu \in\{0,1,2, \cdots\}, i=1,2
$$

Then the Main theorem holds also for the equation $L u=0$.

\subsection{Algebra of Operators in $\mathscr{L}^{m, r, \sigma}$}

Proposition 5.2. The following (a) and (b) hold.

(a) If $0 \leq s \leq r, L \in \mathscr{L}^{m, r, \sigma}$ and $L^{\prime} \in \mathscr{L}^{m-s, r-s, \sigma}$, then

$$
L+L^{\prime} \in \mathscr{L}^{m, r, \sigma}, I_{L+L^{\prime}}(\lambda)=I_{L}(\lambda)+I_{L^{\prime}}(\lambda) .
$$

(b) If $L \in \mathscr{L}^{m, r, \sigma}$ and $L^{\prime} \in \mathscr{L}^{m^{\prime}, r^{\prime}, \sigma}$, then

$$
L L^{\prime} \in \mathscr{L}^{m+m^{\prime}, r+r^{\prime}, \sigma}, \quad I_{L L^{\prime}}(\lambda)=I_{L}(\lambda) I_{L^{\prime}}(\lambda) .
$$

Proof. (a) is trivial. So we prove (b). Consider the following four terms.

$$
\begin{aligned}
& A 1=a_{s}(z)\left(z_{1} D_{1}\right)^{r-s} D_{n}^{m-r} \widetilde{a_{s^{\prime}}}(z)\left(z_{1} D_{1}\right)^{r^{\prime}-s^{\prime}} D_{n}^{m^{\prime}-r^{\prime}} \\
& A 2=a_{s}(z)\left(z_{1} D_{1}\right)^{r-s} D_{n}^{m-r} \widetilde{b_{\beta}}(z) z_{1}^{l(\beta)} D^{\beta} \\
& A 3=b_{\alpha}(z) z_{1}^{l(\alpha)} D^{\alpha} \widetilde{a_{s^{\prime}}}(z)\left(z_{1} D_{1}\right)^{r^{\prime}-s^{\prime}} D_{n}^{m^{\prime}-r^{\prime}} \\
& A 4=b_{\alpha}(z) z_{1}^{l(\alpha)} D^{\alpha} \widetilde{b_{\beta}}(z) z_{1}^{l(\beta)} D^{\beta}
\end{aligned}
$$

where $\alpha \in A(m-s)$ and $\beta \in A\left(m^{\prime}-s^{\prime}\right)$.

First, remark 


$$
\begin{aligned}
\left(z_{1} D_{1}\right)^{k}(a u) & =\sum_{j=0}^{k} C_{j}^{k}\left\{\left(z_{1} D_{1}\right)^{j} a\right\}\left(z_{1} D_{1}\right)^{k-j} u \\
\left(z_{1} D_{1}\right)^{k} & =\sum_{j=1}^{k} c_{k j} z_{1}^{j} D_{1}^{j}
\end{aligned}
$$

where $C_{j}^{k}$ denote the binomial coefficients and $c_{k j}$ is the sequence defined by $c_{k+1, i}=i c_{k i}+c_{k, i-1}$ with $c_{k 1}=c_{k k}=1$ for all $k \geq 1$.

It is then easy to see that $A 1$ can be written as

$$
A 1=\sum_{i} a_{i 0}(z)\left(z_{1} D_{1}\right)^{r^{\prime \prime}-s^{\prime \prime}-i} D_{n}^{m^{\prime \prime}-r^{\prime \prime}}+\sum_{j \geq 1}^{r^{\prime \prime}-s^{\prime \prime}} \sum_{q \geq 0} a_{q j}(z) z_{1}^{q} D_{1}^{q} D_{n}^{m^{\prime \prime}-r^{\prime \prime}-j}
$$

where $m^{\prime \prime}=m+m^{\prime}, r^{\prime \prime}=r+r^{\prime}, s^{\prime \prime}=s+s^{\prime}, a_{00}=a_{s} \widetilde{a_{s^{\prime}}}, a_{i 0}=O\left(z_{1}\right)$ as $z_{1} \rightarrow 0$ for $i \geq 1$.

Moreover, since order $\left(D_{1}^{q} D_{n}^{m^{\prime \prime}-r^{\prime \prime}-j}\right)=m^{\prime \prime}-s^{\prime \prime}-t$ with $t=r^{\prime \prime}-s^{\prime \prime}+j-q$ and $\sigma q$ $+(1-\sigma) q=q>q-j=r^{\prime \prime}-s^{\prime \prime}-t$, we see $A 1$ belongs to the class $\mathscr{L}^{m^{\prime \prime}-s^{\prime \prime}, r^{\prime \prime}-s^{\prime \prime}, \sigma}$ and $I_{A 1}=a_{s}(0) \tilde{a_{s^{\prime}}}(0) \lambda^{r^{\prime \prime}-s^{\prime \prime}}$.

It is also easy to verify that $A 2$ can be written as

$$
A 2=\sum_{p, q} a_{p q}(z) z_{1}^{\gamma-s-q+l(\beta)} D_{1}^{r-s-q} D_{n}^{m-r-p} D^{\beta}
$$

Since order $\left(D_{1}^{r-s-q} D_{n}^{m-r-p} D^{\beta}\right)=m^{\prime \prime}-s^{\prime \prime}-t$ with $t=p+q$ and

$$
\sigma\{r-s-q+l(\beta)\}+(1-\sigma)\left(r-s-q+\beta_{1}\right) \geq r^{\prime \prime}-s^{\prime \prime}-t,
$$

we see $A 2$ belongs to $\mathscr{L}^{m^{\prime \prime}-s^{\prime \prime}, r^{\prime \prime}-s^{\prime \prime}, \sigma}$. It is clear that $I_{A 2}=0$.

$A 3$ can be written as

$$
A 3=\sum_{\widetilde{\alpha}, p, q} a_{\widetilde{\alpha}, \cdot q}(z) z_{1}^{r^{\prime}-s^{\prime}-p-q+l(\alpha)} D^{\alpha-\widetilde{\alpha}} D_{1}^{\gamma^{\prime}-s^{\prime}-p} D_{n}^{m^{\prime}-r^{\prime}}
$$

with $q \leq \widetilde{\alpha}_{1}$. Since order $\left(D^{\alpha-\widetilde{\alpha}} D_{1}^{r^{\prime}-s^{\prime}-p} D_{n}^{m^{\prime}-r^{\prime}}\right)=m^{\prime \prime}-s^{\prime \prime}-t$ with $t=|\widetilde{\alpha}|+p$ and

$$
\sigma\left(r^{\prime}-s^{\prime}-p-q+l(\alpha)\right)+(1-\sigma)\left(\alpha_{1}-\widetilde{\alpha}_{1}+r^{\prime}-s^{\prime}-p\right) \geq r^{\prime \prime}-s^{\prime \prime}-t,
$$

the term $A 3$ also belongs to $\mathscr{L}^{m^{\prime \prime}-s^{\prime \prime}, r^{\prime \prime}-s^{\prime \prime}, \sigma}$.

$A 3$ may contain some terms with form $a(z)\left(z_{1} D_{1}\right){ }^{p} D_{n}^{m+m^{\prime}-r-r^{\prime}}$. Let $\alpha \in A$ (m $-s)$ with $\alpha=\left(\alpha_{1}, \alpha^{\prime \prime}, m-r\right)$. Since $\alpha^{\prime \prime} \neq 0$, we have $\alpha_{1}<r-s$. When $\sigma=0$, there are no such $\alpha$, because $\sigma l(\alpha)+(1-\sigma) \alpha_{1}=\alpha_{1} \geq r-s$. When $\sigma>0$, we have $\sigma\left(l(\alpha)-\alpha_{1}\right) \geq r-s-\alpha_{1}>0$ and consequently $l(\alpha)>\alpha_{1}$. Therefore, even if there are such terms, it holds that $a(z)=O\left(z_{1}\right)$ as $z_{1} \rightarrow 0$. Hence $I_{A 3}=0$.

$A 4$ can be written as

$$
A 4=\sum_{\widetilde{\alpha}} a_{\alpha \widetilde{\alpha}}(z) z_{1}^{l(\alpha)+l(\beta)-\widetilde{\alpha}_{1}} D^{\alpha+\beta-\widetilde{\alpha}}
$$


Since $\operatorname{order}\left(D^{\alpha+\beta-\tilde{\alpha}}\right)=m^{\prime \prime}-s^{\prime \prime}-t$ with $t=|\widetilde{\alpha}|$ and

$$
\sigma\left(l(\alpha)+l(\beta)-\widetilde{\alpha}_{1}\right)+(1-\sigma)\left(\alpha_{1}+\beta_{1}-\widetilde{\alpha}_{1}\right) \geq r^{\prime \prime}-s^{\prime \prime}-t,
$$

$A 4$ belongs to $\mathscr{L}^{m^{\prime \prime}-s-s^{\prime}, r^{\prime \prime}-s-s^{\prime}}$ and $I_{A 4}=0$.

Now that $L L^{\prime}$ is the sum of such terms as $A 1, A 2, A 3$ and $A 4$, by using (a) one can see (b) immediately.

(Q.E.D.)

When $r=0$, the class $\mathscr{L}^{m, 0, \sigma}$ is free from $\sigma$, and so one may simply write $\mathscr{L}^{m, 0}$. Every operator $Q(z, D)$ of order $\mathrm{m}$ with holomorphic coefficients belongs to $\mathscr{L}^{m, 0}$.

Corollary 5.3. Let $P \in \mathscr{L}^{2,1, \sigma}$ and $Q_{i} \in \mathscr{L}^{i, 0}$. Then the operator $L$ defined by (19) belongs to $\mathscr{L}^{2 r, r, \sigma}$ and

$$
I_{L}(\lambda)=I_{P}^{r}(\lambda)+Q_{1}^{\circ}(0, \nu) I_{P}^{r-1}(\lambda)+\cdots+Q_{r}^{\circ}(0, \nu)
$$

where $\nu=(0, \cdots, 0,1) \in \mathbb{C}^{n}$.

\subsection{Proof of Theorem 5.1}

As we have observed in the preceding section, by appropriate change of variables reducing one of $K^{i}$ to $z_{n}=0$, the operator $P$ belongs to $\mathscr{L}^{2,1, \sigma}$ with $\sigma=0$, $1 / 2$ or 1 . Besides, the operator $L$ conserves its form. Therefore $L$ belongs to $\mathscr{L}^{2 r, r, \sigma}$ by Corollary 5.3 .

Since $\zeta_{1}=\lambda^{i}\left(z, \zeta^{\prime}\right)$ corresponds to $\eta_{1}=\mu^{i}\left(w, \eta^{\prime}\right)$ and $\mu^{i}\left(0, w^{\prime}, \nu^{\prime}\right)=0$, we see $C_{k}^{i}$ are invariant. It means $I_{L}(\mu)=G^{i}(\mu)$.

Noting the above, one can prove this theorem in the same way as the proof of Main theorem.

(Q.E.D.)

\section{References}

[1] Alinhac, S., Branching of singularities for a class of hyperbolic operators, Indiana U. Math. J., 27 (1978), 1027-1037.

[2] Fujiie, S., Singular Cauchy problems of higher order with characteristic initial surface, $J$. Math. Kyoto Univ., 33 (1993), 1-27.

[3] Grauert, H. and Fritszche, K., Several Complex Variables, Springer, Berlin, 1976.

[4] Hamada, Y., Leray, J. et Wagschal, C., Systéme d'équations aux dérivées partielles à caractéristiques multiples; problème de Cauchy ramifié; hyperbolicité partielles, J. Math. Pures Appl., 55 (1976), 297-352.

[5] Hanges, N., Parametrics and propagation of singularities for operators with non-involutive characteristics, Indiana U. Math. J., 28(1979), 87-97.

[6] Hasegawa, Y., On the initial value problems with data on a characteristic hypersurface, $J$. Math. Kyoto Univ., 13 (1973), 579-593.

[7] Igari, K., Characteristic Cauchy problems and analytic continuation of holomorphic solutions, 
Ark. Mat., 28 (1990), 289-300.

[8] lgari, K., Fuchs type localizations and non-existence of singular solutions, Funkcial. Ekvac., 37 (1994), 537-547.

[9] On the branching of singularities in complex domains, Proc. Japan Acad. Ser. A, 70 (1994), 128-130.

[10] Leray, J., Uniformisation de la solution du problème linéaire analytique de Cauchy près de la variété qui porte les données de Cauchy (Problème de Cauchy I), Bull. Soc. Math. France, 85 (1957), 389-429.

[11] Oaku, T., A canonical form of a system of microdifferential operators with non-involutory characteristics and branching of singularities, Invent. Math., 65 (1982), 491-525.

[12] Ouchi, S., Singularities of solutions of equations with non-involutive characteristics- I ; the case of second order Fuchsian equations. J. Math. Soc. Japan, 45 (1993), 215-251.

[13] Perssson. J., Ramification of the solutions of the Cauchy problem for a special second order equation with singular data, Comm. P. D. E., 17 (1992), 23-31.

[14] Schiltz, D., Vaillant, J. et Wagschal, C., Problème de Cauchy ramifié: racine caractéristique double ou triple en involution, J. Math. Pures Appl., 4 (1982), 423-443.

[15] Urabe, J., Hamada's theorcm for a certain type of operators with double characteristics, $J$. Math. Kyoto Univ., 23 (1983), 301-339.

[16] Wagschal, C., Problème de Cauchy ramifié: racine caractéristique multiples holomorphes de multiplicité variable, J. Math. Pures Appl., 62 (1983), 99-127.

[17] _ Problème de Cauchy ramifié pour une classe d'opérateurs à caractéristiques tangentes (I), J. Math. Pures Appl., 67 (1988), 1-21.

[18] Zerner, M., Domaines d'holomorphie des fonctions vérifiant une équation aux dérivées partielles, C. R. Acad. Sci. Paris, 272 (1971), 1646-1648. 
